\title{
Intraoperative Ultrasound Guidance for Transanal Endoscopic Microsurgery
}

Philip Pratt, Aimee Di Marco, Christopher Payne, Ara Darzi, and Guang-Zhong Yang

Hamlyn Centre for Robotic Surgery

Imperial College of Science, Technology and Medicine

London SW7 2AZ, UK

\{p.pratt, a.di-marco, cjp04,a.darzi,g.z.yang\}@imperial.ac.uk

\begin{abstract}
Local excision of rectal cancer with transanal endoscopic microsurgery has proved to be a viable alternative to conventional, more radical techniques, but the reduced sensory experience presents significant challenges for the surgeon. Accurate identification and complete removal of lesions and subsurface targets is currently a difficult task, often exacerbated by intraoperative tissue deformation. This work describes novel ultrasound calibration and effective visualisation methods designed to meet these requirements, relying solely on optical measurements and pattern tracking. Detailed quantitative phantom and porcine validation experiments confirm that the technique is both practical and an accurate means for assessing lesion thickness intraoperatively, leading directly to human clinical trials.
\end{abstract}

\section{Introduction}

Rectal carcinoma accounts for a significant number of cancer-related deaths in the developed world. Transanal endoscopic microsurgery [1] was introduced as an alternative to conventional, more radical resection techniques for the treatment of earlystage lesions, with the goal of reducing the relatively high mortality and morbidity, including functional impairment and stoma. Indeed, the recent prevalence of cancer screening programmes demands the more widespread use of an effective minimally invasive technique, capable of proportionately safe and accurate resection. The goal of this work is to provide real-time, intraoperative ultrasound guidance using a small microsurgery transducer, such that its images are correctly registered to and fused with endoscopic views of the surgical scene. Moreover, by addressing some of the remaining barriers that typically impede use in human subjects, the quantitative phantom and porcine study presented here suggests a rapid path for clinical translation.

Safety and convenience, combined with recent advances in image quality, have made ultrasound images an attractive candidate for use in augmented reality systems designed for diagnosis and intervention. This is particularly true in the context of minimally invasive, including robotic, surgical procedures where the reduced sensory experience presents the surgeon with significant challenges. Notable advances in this area include the 'sonic flashlight' of Stetten et al. [2], the 'DaVinci Canvas' developed by Leven et al. [3], the technique for freehand 3D ultrasound described by Ali and Logeswaran [4], 
and applications during laparoscopic partial nephrectomy and bronchoscopy introduced by Cheung et al. [5] and Dressel et al. [6], respectively. Langø et al. [7] provide an extensive review of navigated laparoscopic ultrasound and describe their own experiences of optimal resection planning during adrenalectomy in a porcine model. In addition, Schneider et al. [8] have performed a feasibility study for 'pick-up' ultrasound, using the renal vasculature to perform registration to preoperative CT images.

However, the relatively small scale afforded by the transanal approach and transducer size dictates that the localisation techniques employed to date in the most relevant studies, namely exogenous electromagnetic or optical tracking, robot kinematic feedback, either individually or in combination, are not sufficiently accurate or practical for the intended application. Indeed, the difficulties associated with performing precise hand-eye and probe calibrations, typically required in a contemporaneous manner, present important barriers to successful clinical translation.

During surgery, the radial extent of lesions is significantly easier to assess than the depth of penetration through the mucosal and sub-mucosal layers of the rectum wall. This can lead to excisions being too deep, resulting in perforation, or too shallow, leading to incomplete margins. The paucity of depth information is apparent in Fig. 1 (left), which shows a typical endoscopic view during a human case immediately prior to resection. Adopting lesion thickness measurement as an exemplar application, this work therefore describes a novel use for ultrasound overlay, based on optical pattern tracking alone, using a new dual pattern calibration method. An efficient tracking algorithm, driven by topological relationships between features, and visualisation methods is described. Phantom and porcine validation experiments are performed to measure overlay accuracy and gauge suitability for clinical translation.

\section{Materials and Methods}

\subsection{Microsurgery Probe Calibration}

The ultrasound transducer used in this study is a UST-533 multi-frequency linear array microsurgery probe (Hitachi Aloka Medical Ltd., Tokyo, Japan), driven by a ProSound ALPHA 10 cart. As illustrated in Fig. 1 (centre), its small dimensions and associated field of view (approximately 60mm) make it an ideal choice given the limited workspace imposed by the transanal endoscopic approach and the intended targets. By selecting a relatively high zoom factor on the cart (1 pixel $\sim 50 \mu \mathrm{m})$, the appropriate balance between level of detail and depth of tissue penetration is struck.
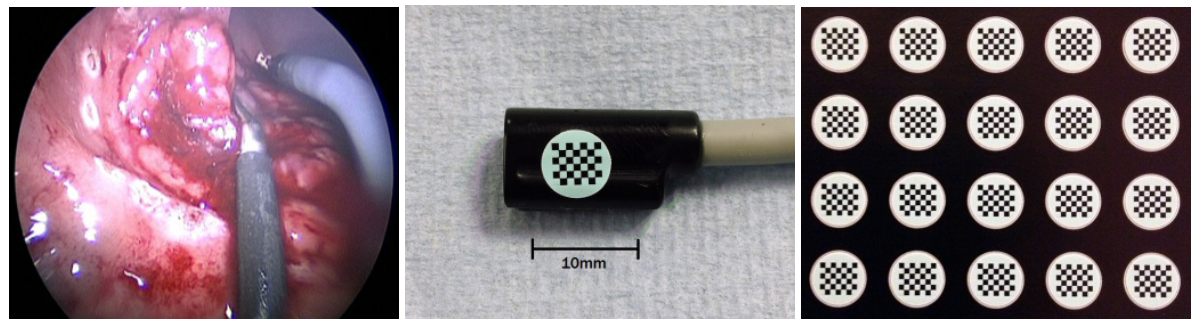

Fig. 1. Endoscopic view prior to resection, microsurgery probe and KeySurgical marker dots 
The probe calibration process seeks to determine the static spatial transformation from the ultrasound image frame to the transducer frame. The latter is defined by a semi-permanently attached KeyDot ${ }^{\circledR}$ marker (KeySurgical Inc., Eden Prairie, MN, USA). Fig. 1 (right) illustrates the design, where non-symmetrical chessboard patterns are laser-engraved on $6.35 \mathrm{~mm}$ diameter, $76.2 \mu \mathrm{m}$ thickness destructible acrylate discs. Overcoming another important translational barrier, the markers and adhesive are approved for human use and can withstand harsh environments and temperatures, including repeated sterilisation cycles. Further promoting longevity, the radial design ensures that there are no corners to lift. Once calibrated, the relationship between image and transducer frames remains fixed until the marker is replaced.

The spatial transformation, represented as an image scaling followed by a 6 DOF translation and rotation, is estimated by scanning a calibration object of known geometry and associating image points and their corresponding spatial positions. Following Chen et al. [9] and other studies, a multiple Z-wire arrangement is employed, where the scan images are manually segmented. The main challenge here is in the accurate manufacture of the phantom at a necessarily small scale. To this end, the calibration phantom is printed using an Objet260 Connex rapid prototype 3D printer (Objet Ltd., Rehovot, Israel). Typical build accuracies are in the range $20-85 \mu \mathrm{m}$.

Fig. 2 (left) shows a cross-section through the phantom design, revealing conical guide holes and the boss on which a calibration pattern is mounted. Chosen for their very high straightness tolerance, Micron Hard high speed steel wires (Nachi-Fujikoshi Corp., Tokyo, Japan), of nominal diameter $250 \mu \mathrm{m}$, are threaded through $300 \mu \mathrm{m}$ diameter holes in the opposing phantom walls. The complete device is shown in Fig. 2 (centre), together with a typical ultrasound scan image (right), the well having been filled with water at a temperature of approximately $38^{\circ} \mathrm{C}$.

Images are captured using a $5 \mathrm{~mm}$ diameter mono laparoscope (Karl Storz $\mathrm{GmbH}$, Tuttlingen, Germany), forming part of the standard equipment inventory. Intrinsic camera parameters and distortion coefficients are estimated using an implementation of Zhang's calibration method [10,12], using at least twenty phantom pattern images at different distances and orientations. This study employs the novel method of capturing both the phantom and transducer patterns in the same image (Fig. 3, left). By recovering the extrinsic parameters comprising the transformations between the patterns and their projections in that image, through the minimisation of reprojection error [12], it is possible to estimate the relationship between the phantom and transducer coordinate systems.
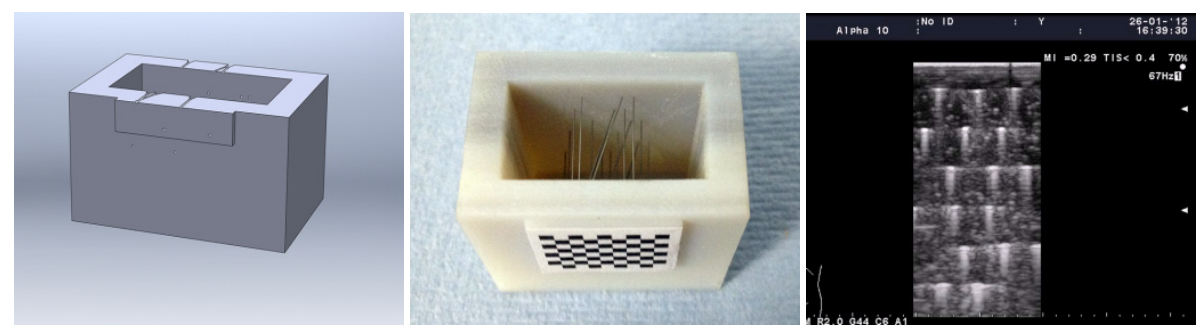

Fig. 2. Calibration phantom section, internal Z-wire pattern and microsurgery probe image 
Specifically, Fig. 3 (centre) illustrates the coordinate frames involved during the calibration procedure: the calibration phantom frame $\mathrm{P}$, the ultrasound image frame $\mathbf{U}$ and the transducer frame T. The camera coordinate frame is not shown. Given 2D points $\mathbf{p}_{i}$ in the ultrasound image frame and corresponding $3 \mathrm{D}$ locations $\mathbf{q}_{i}$ in the calibration phantom frame, the desired scale factor $s$ and transformation $\mathbf{M}_{U T}$, mapping from the ultrasound image frame to the transducer frame, are found by minimisation of the following expression.

$$
\sum_{i}\left\|\mathbf{M}_{U T} \cdot s \cdot \mathbf{p}_{i}-\mathbf{E}_{T}^{-1} \mathbf{E}_{P} \cdot \mathbf{q}_{i}\right\|^{2}
$$

The matrices $\mathrm{E}_{T}$ and $\mathrm{E}_{P}$ represent the transducer and phantom extrinsics, respectively. Formulated as an absolute orientation problem [11], the solution can be found in closed form. The scale factor $s$ is determined as the ratio of the root mean-squared deviations of the coordinates from their respective centroids. For comparison, the expression is also minimised in a more general form where the $\mathrm{X}$ and $\mathrm{Y}$ image scale factors are allowed to vary independently. Since the image-to-probe transformation is independent of the camera and associated video hardware, probe calibrations performed at different resolutions are, in principle, interchangeable once an appropriate ultrasound image scaling and translation has been applied. In this study, calibrations are performed at both SD $(720 \times 576 \mathrm{i})$ and HD $(1920 \times 1080 \mathrm{i})$ resolution to determine whether any improvement in accuracy using the latter is sufficient to warrant the inevitable decrease in processing performance.
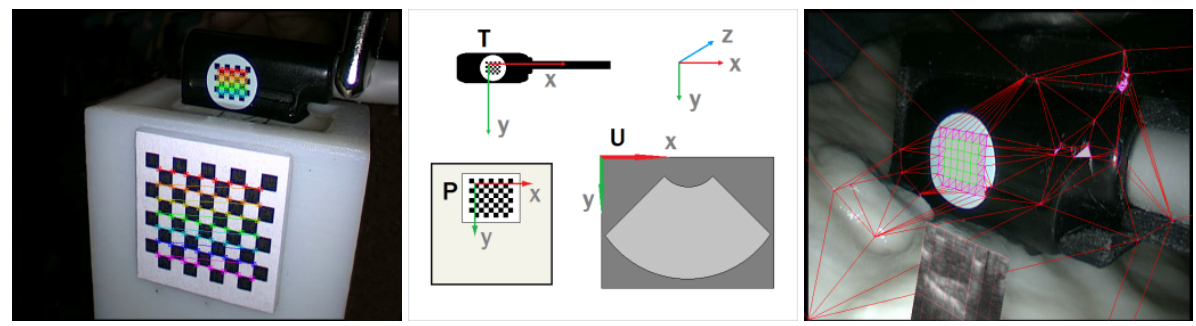

Fig. 3. Dual chessboard pattern recovery, coordinate frames and pattern tracking with overlay

\subsection{Real-Time Pattern Tracking}

While contour-based approaches to chessboard tracking [12] are typically robust, this study builds on the work of Shu et al. [13] and adopts a more efficient method, suitable for real-time use, based on Delaunay triangulation and the exploitation of geometric and topological constraints imposed by the pattern. Each frame, captured with a Quadro SDI card (NVIDIA Corp., Santa Clara, CA, USA), is processed as follows:

- feature selection (i.e. Sobel filter, Eigenimage generation, thresholding) [12]

- sub-pixel corner refinement [12] and averaging of corners in close proximity

- Delaunay triangulation [12]

- elimination of triangles with edge length greater than specified threshold

- elimination of triangles where edge ratio metric is greater than specified limit

- elimination of triangles that do not have three neighbours 
- conversion to quadrilaterals by comparison and pairing of longest edges

- vertex orientation using cross products

- recursive flood-fill to determine relationships between quadrilaterals

- trim quadrilaterals that have fewer than two neighbours

- select largest group of connected quadrilaterals then locate 'top left' corner

- check width and height of connected quadrilateral pattern

- determine pattern orientation by comparing pixel averages in opposing corners.

The optimal tracking parameters (e.g. corner quality threshold) are determined by empirical optimisation over archetypal footage. In the event of a recognition failure, the last good pattern is used for up to a maximum of eight frames. Fig. 3 (right) shows the triangulation and quadrilaterals comprising a successful recognition.

\subsection{Visualisation}

Once a valid pattern has been identified, the corners and prevailing camera parameters are used to find the optimal extrinsic transformation [12]. Concatenated with the calibrated transformation $\mathbf{M}_{\mathrm{UT}}$ this is used subsequently to project and render the ultrasound image texture in the correct location. The texture is blended with the underlying scene, using an adjustable alpha coefficient. The user may move the crop boundaries applied to the image texture, and also enable a measurement grid overlay with $1 \mathrm{~mm}$ and $5 \mathrm{~mm}$ graduations (Fig. 4, left). Textures and extrinsics are stored in a GPU ring buffer, such that 3D freehand reconstructions from multiple slices can be made at any time (Fig. 4, centre). Furthermore, a custom pixel shader is used to pass the ultrasound image through a 1D transfer function performing histogram equalisation on-the-fly. This markedly improves image contrast and thus interpretation of structure.
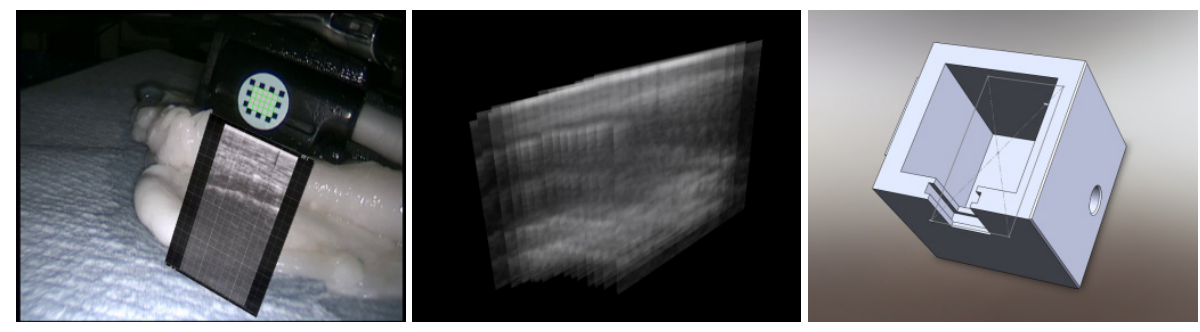

Fig. 4. Overlay ruler markings, freehand 3D reconstruction and validation phantom design

\subsection{Validation}

The first validation stage aims to measure ultrasound overlay accuracy and the extent of the operational envelope defined by probe angle of incidence and laparoscope proximity. The original calibration phantom was modified to support a single crosswire feature (Fig. 4, right). Direct line of sight was achieved by sliding out its removable door and removing the plug, thereby draining the water bath. Once the two image features representing the wires are made coincident in the ultrasound scan, overlay accuracy can be assessed by superimposing the overlay and undistorted direct view of the wire crossing point (Fig. 5, left). 

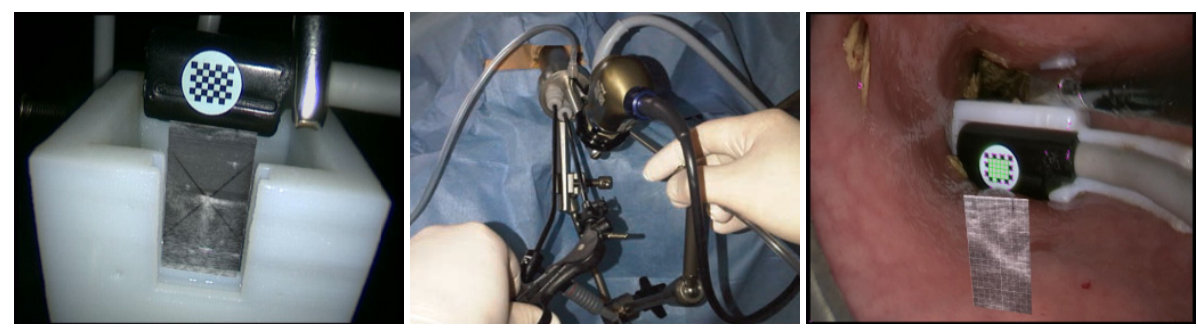

Fig. 5. Validation phantom superimposition, equipment setup and in vivo porcine study

Thereafter, intact ex vivo porcine rectum samples were used to assess the accuracy of lesion thickness measurement. The microsurgery probe was mounted in a customdesigned holder and wire guide, with exposed foot to facilitate secure mating with the grasping instrument. In each sample, six simulated silicone lesions of varying thicknesses (from $1.5 \mathrm{~mm}$ to $4 \mathrm{~mm}$ ) were inserted between tissue layers. This material was chosen as it exhibits imaging responses similar to those observed during preoperative examination. Six subjects (five surgeons, one engineer) were recruited to the study and asked to estimate lesion thickness using ultrasound overlay. Thickness estimates were compared against subsequent caliper measurements.

Finally, an in vivo porcine trial was conducted to confirm that the system as a whole is sufficiently practical and robust in a live theatre environment. Following proctoscope and instrument insertion (Fig. 5, centre), pneumorectum was established. Although there was no specific pathology to investigate, surveys of rectum wall thickness were made with a view, in practice, of reducing the chance of perforation. This is of relevance to any lesion above the peritoneal reflection, and is of particular importance in human female subjects with lesions situated in the anterior wall, where accidental creation of a rectovaginal fistula must be avoided.

\section{Results}

From a series of eight separate calibration runs at SD resolution, the mean, standard deviation and maximum of the residual errors were found to be $206 \mu \mathrm{m}, 53 \mu \mathrm{m}$ and $277 \mu \mathrm{m}$, respectively. Employing the most accurate of these calibrations, the corresponding results for ultrasound image and direct sight superimposition, gathered over a range of eight different camera poses and transducer positions, were found to be $669 \mu \mathrm{m}, 255 \mu \mathrm{m}$ and $961 \mu \mathrm{m}$. The calibrations performed at HD resolution did not result in a significant residual error improvement. Similarly, the use of a general transformation (i.e. separate scaling in the $\mathrm{X}$ and $\mathrm{Y}$ directions) resulted in only a $23 \mu \mathrm{m}$ improvement in residual error. The relative size of the validation error in comparison to the residuals can be attributed to the difficulty in achieving an accurate cross-wire image feature alignment when configuring the phantom and probe.

Table 1. Probe tracking operational envelope

\begin{tabular}{c|ccc} 
& range & $\min$ & $\max$ \\
\hline rotate $\mathrm{X}$ & $97^{\circ}$ & $-44^{\circ}$ & $53^{\circ}$ \\
rotate $\mathrm{Y}$ & $102^{\circ}$ & $-54^{\circ}$ & $48^{\circ}$ \\
translate $\mathrm{Z}$ & $42 \mathrm{~mm}$ & $22 \mathrm{~mm}$ & $64 \mathrm{~mm}$ \\
\hline
\end{tabular}


Table 1 shows a typical measurement of the probe tracking algorithm's operational envelope, i.e. the range of probe positions resulting in consistently successful pattern recognitions, where a perpendicular arrangement of probe and endoscope defines the rotation origins, and the $\mathrm{Z}$ translation is measured from endoscope tip to pattern centre. It can be seen that there is a relatively wide operating envelope, although at high illumination levels, performance was observed to deteriorate in a region close to the rotational origins. This is due to the intensity of reflections and blooming in the camera sensor. The design of the probe mounting's angled foot ensures that this region is avoided in practice, while offering significant help in maintaining wide overlay areas.

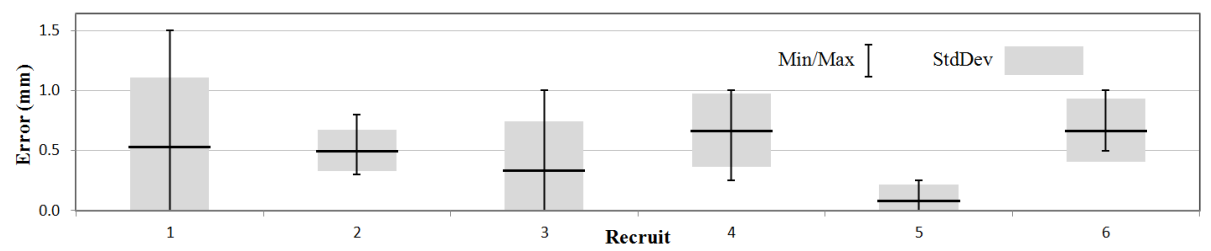

Fig. 6. Mean ex vivo lesion thickness measurement errors

Mean lesion thickness measurement errors for each recruit assessed on the ex vivo model are shown in Fig. 6. The overall mean error is $0.46 \mathrm{~mm}$, showing an admissible result given typical sizes $(9-40 \mathrm{~mm})$ of transanally resected lesions. Median responses to the study questionnaire indicate that integrated ultrasound image overlay makes depth measurement much easier and convenient than use of the cart separately, that it helps convey a relative sense of scale, and that the inevitable small video delay is not noticeable. Fig. 5 (right) shows a typical snapshot from the in vivo porcine trial. This exercise highlighted the importance of selecting optimal illumination levels.

\section{Discussion and Conclusion}

In common with most camera-based systems, this work has the limitation that it cannot automatically adjust to changes in zoom or focus position. Although, crucially, no probe re-calibration is required in this event, the current setup still demands a repeat camera calibration. To make this practical for intraoperative use, the system will be extended to support zoom position interpolation over a discrete space of precalibrated camera intrinsic parameters. Much like the procedure itself, the restricted workspace and colinearity of instruments enforced by the transanal approach make probe manipulation a challenge, but the planned development and integration of a transanal robotic device will facilitate large-scale survey of the entire lumen. However, even with an accurate sub-surface measurement capability, this does not in itself guarantee completely negative radial and deep margins. Future integration of probebased confocal laser endomicroscopy will allow immediate post-excision inspection.

Procedurally, transanal endoscopic microsurgery has not changed significantly since its original introduction in the 1980s. Through the addition of live ultrasound overlay, this work represents a significant innovation, required in part to meet the growing demands of prevalent cancer screening programmes. Through the introduction of novel calibration and tracking techniques, detailed calibration and validation 
results show sufficient levels of measurement accuracy, repeatability, computational efficiency and robustness in the context of realistic scenarios. In combination with medically-approved materials and transducers, and the fact that preoperative workup includes transanal endosonography examination in which rectal wall lesions are initially diagnosed and staged, these prepare the way for immediate human clinical translation. Naturally, the methods described in this work are also directly applicable to other minimally invasive procedures, including robotically-assisted interventions.

\section{References}

1. Cataldo, P., Buess, G.: Transanal Endoscopic Microsurgery: Principles and Techniques. Springer (2009)

2. Stetten, G., Chib, V., Hildebrand, D., Bursee, J.: Real time tomographic reflection: Phantoms for calibration and biopsy. In: IEEE/ACM International Symposium on Augmented Reality, pp. 11-19 (2001)

3. Leven, J., Burschka, D., Kumar, R., Zhang, G., Blumenkranz, S., Dai, X., Awad, M., Hager, G., Marohn, M., Choti, M., Hasser, C., Taylor, R.: DaVinci Canvas: A Telerobotic Surgical System with Integrated, Robot-Assisted, Laparoscopic Ultrasound Capability. In: Duncan, J.S., Gerig, G. (eds.) MICCAI 2005, Part I. LNCS, vol. 3749, pp. 811-818. Springer, Heidelberg (2005)

4. Ali, A., Logeswaran, R.: A visual probe localization and calibration system for cost-effective computer-aided 3D ultrasound. Computers in Biology and Medicine 37, 1141-1147 (2007)

5. Cheung, C.L., Wedlake, C., Moore, J., Pautler, S.E., Peters, T.M.: Fused Video and Ultrasound Images for Minimally Invasive Partial Nephrectomy: A Phantom Study. In: Jiang, T., Navab, N., Pluim, J.P.W., Viergever, M.A. (eds.) MICCAI 2010, Part III. LNCS, vol. 6363, pp. 408-415. Springer, Heidelberg (2010)

6. Dressel, P., Feuerstein, M., Reichl, T., Kitasaka, T., Navab, N., Mori, K.: Direct Co-calibration of Endobronchial Ultrasound and Video. In: Liao, H., Eddie Edwards, P.J., Pan, X., Fan, Y., Yang, G.-Z. (eds.) MIAR 2010. LNCS, vol. 6326, pp. 513-520. Springer, Heidelberg (2010)

7. Langø, T., Vijayan, S., Rethy, A., Vâpenstad, C., Solberg, O.V., Mârvik, R., Johnsen, G., Hernes, T.: Navigated laparoscopic ultrasound in abdominal soft tissue surgery: technological overview and perspectives. International Journal of Computer Assisted Robotics and Surgery (2011)

8. Schneider, C., Guerrero, J., Nguan, C., Rohling, R., Salcudean, S.: Intra-operative "PickUp" Ultrasound for Robot Assisted Surgery with Vessel Extraction and Registration: A Feasibility Study. In: Taylor, R.H., Yang, G.-Z. (eds.) IPCAI 2011. LNCS, vol. 6689, pp. 122-132. Springer, Heidelberg (2011)

9. Chen, T., Thurston, A., Ellis, R., Abolmaesumi, P.: A real-time freehand ultrasound calibration system with automatic accuracy feedback and control. Ultrasound in Medicine and Biology 35(1), 79-93 (2009)

10. Zhang, Z.: A flexible new technique for camera calibration. IEEE Transactions on Pattern Analysis and Machine Intelligence 22(11), 1330-1334 (2000)

11. Horn, B.: Closed-form solution of absolute orientation using unit quaternions. Journal of the Optical Society of America A 4(4), 629-642 (1987)

12. Bradski, G., Kaehler, A.: Learning OpenCV: Computer Vision with the OpenCV Library. O'Reilly Media, Inc. (2008)

13. Shu, C., Brunton, A., Fiala, M.: A topological approach to finding grids in calibration patterns. Machine Vision and Applications 21, 949-957 (2010) 